\title{
Behavioral and electrophysiological evidence for cortical reorganization of function in rats with serial lesions of the visual cortex
}

\author{
S. W. SCHEFF and D. C. WRIGHT \\ Department of Psychology and Dalton Research Center, University of Missouri, Columbia, Missouri 65201
}

\begin{abstract}
Male albino rats relearned a black-white discrimination for food reward in a T-maze following bilateral removal of posterior neocortex. Single-stage-lesion animals were given a bilateral lesion in one operation and also underwent a sham operation. These were given in the order: lesion-sham ("early") or sham-lesion ("late"). Training on the task was interpolated between operations. Multiplestage-lesion animals suffered unilateral lesions in each of two operations. Only half of these animals received interpolated training. Animals given single-stage lesions, whether "early" or "late," made as many errors in relearning as they had in original, preoperative, learning. Animals given multiplestage lesions but no interoperative training also showed no savings in relearning. Animals given multiple-stage lesions and training between operations made fewer errors in relearning than in original learning, i.e., showed positive savings. After behavioral testing, cortex contiguous with the posterior lesion was mapped for evoked responses to light flash under chloralose anesthesia. The electrophysiological responsiveness of contiguous cortex was quantitatively and qualitatively different in animals that had received multiple stage lesions and interoperative training. Cortical reorganization may be uniquely important following multiple stage lesions with interoperative training.
\end{abstract}

Rats trained on a two-choice brightness discrimination and then subjected to single-stage (SS) bilateral removal of the visual cortex show no savings in postoperative relearning of the task, that is, they require as many trials to relearn the task as they required to learn it originally (Lashley, 1920, 1922, 1926, 1929, 1930). In contrast, rats trained on a brightness task and given two separate unilateral lesions of the visual cortex show positive savings in relearning (fewer trials to relearn than to learn) if they are given additional training trials during the interval between the two unilateral lesions, but show no savings following multiple-stage (MS) lesions if no training is given in the interoperative interval (IOI) (Glendenning, 1972; Kircher, Braun, Meyer, \& Meyer, 1970; Petrinovich \& Carew, 1969; Thompson, 1960).

Rats given SS lesions of the visual cortex cannot learn or relearn a pattern discrimination (Horel, Bettinger, Royce, \& Meyer, 1966; Lashley, 1929; Spear \& Barbas, 1975). Rats given MS lesions and training during the IOI can relearn a pattern discrimination (Spear \& Barbas, 1975). Moreover, rats given MS lesions and merely allowed to freely explore a patterned visual environment during the IOI

Supported in part by the Dalton Research Center, University of Missouri-Columbia. The authors thank Dr. J. D. Dexter for the loan of equipment and for his encouragement and advice. S. W. Scheff is now at the Department of Psychobiology, University of California, Irvine, California, Irvine 92717. can relearn a pattern discrimination, but MS animals that lack such experience cannot (Dru, Walker, \& Walker, 1975).

Although they have suffered equivalent visual cortex damage, MS animals with appropriate IOI experience behave very differently from SS animals and MS animals that lack appropriate IOI experience. This is the "serial lesion effect."

In a previous study (Scheff, Wright, Morgan, \& Powers, 1976), rats relearned a black-white discrimination after SS or MS visual cortex lesion. The animals were then given bilateral lesions of either cortex contiguous with the visual cortex lesion site (C) or an equivalent volume of cortex noncontiguous with the visual cortex lesion (NC), and were again retrained on the task. In second retraining, SS animals with $\mathrm{C}$ lesions did not differ from SS animals with NC lesions and both groups showed positive savings. The MS animals with NC lesions did not differ from either of the SS groups, but MS animals with $\mathrm{C}$ lesions were inferior to all groups and showed negative savings in second retraining. In short, the effects of $\mathrm{C}$ and $\mathrm{NC}$ lesions were equivalent among animals that had "recovered" from SS visual cortex lesions but markedly different among MS animals.

Appropriate IOI experience and the integrity of cortex contiguous with the visual cortex appear to be necessary for the behavioral manifestation of the serial lesion effect. The present study was designed to look for electrophysiological evidence that cortical reorganization plays a role in that effect. 


\section{METHODS}

\section{Subjects}

Forty-five Sprague-Dawley-derived male albino rats (Holtzman, Madison, Wisconsin) were used. The animals were 75-90 days old at the start of the investigation and were housed singly in metal cages under a $12 / 12 \mathrm{~h}$ light-dark cycle. Water was available ad lib., although food (Purina Rat Chow) was restricted to two large pellets (average $8 \mathrm{~g} /$ pellet) per day, given immediately after training.

\section{Apparatus}

A T-maze, as described in Scheff et al. (1976), was used. The painted steel back wall of the maze arms provided either a white or a black painted surface that extended from the choice point to the offset goal areas at the end of each arm. Containers of wet mash (Purina Rat Chow and water) were placed in both goal areas to equate odor cues, but the container in the goal area at the end of the incorrect arm was covered with wire screen to prevent access to the mash.

\section{Procedure}

Four days before the start of pretraining, the subjects were handled for 10 min each day. For half of the subjects, black was designated as the correct stimulus, and half the subjects were run with white correct. On Day 1 of pretraining, they were run on a series of five forced trials to the correct side which always appeared on the left. On each trial, the animal was placed in the startbox with the opaque guillotine door open, permitting the animal to see the stimuli. After $10 \mathrm{sec}$, the clear Plexiglas door was opened, permitting the animals to move down the runway towards the stimulus plates. The subject was given 2 min to enter an arm of the maze. The animal was then allowed $10 \mathrm{sec}$ access to the mash in the goal area and was returned to the startbox to await the start of the next trial.

Day 2 of pretraining was exactly like Day 1, except that the correct stimulus always appeared on the right side.

On Day 3, the rats were run 10 forced trials with the position of the correct stimulus varied according to a Gellermann (1933) sequence. During this pretraining session, the animal remained in the startbox for $15 \mathrm{sec}$ with the Plexiglas door closed. After $10 \mathrm{sec}$, the opaque door was opened and the animal was permitted to view the discriminanda for $5 \mathrm{sec}$, at which time the clear Plexiglas door was opened and the animal was permitted to move toward the arms of the maze. All other timing parameters were as on Days 1 and 2.

Day 4 began the start of the training period with 10 trials per day. On each trial, the animal was placed in the startbox, and after $10 \mathrm{sec}$, the opaque guillotine door separating the runway from the arms of the maze was opened. Five seconds later, the clear Plexiglas door was raised and the animal then had 2 min to choose one of the two maze arms. Following choice (the animal's body completly in one arm), the opaque guillotine door was closed and the animal remained in the chosen arm/goal area for $10 \mathrm{sec}$. The right and left position of the correct arm varied according to a Gellerman (1933) sequence. The animals were run 10 noncorrection trials per day until they achieved a criterion of 9 out of 10 correct on 2 successive days.

Table 1 provides an outline of the experimental design. The animals were matched on the basis of trials to criterion in original training and 9 animals were assigned to each of the five surgical groups. All animals received two separate operations given 9 days apart. Group Sham underwent two sham operations. The single-stage-lesion groups received one sham operation and one operation in which a bilateral visual cortex lesion was made. In the single-stage "early" (SSE) group, the lesion was made in the first operation. For the single-stage "late" (SSL) group, the sham operation was first. For the multiple-stage-lesion groups, bilateral visual cortex removal was accomplished by administering unilateral lesions in each of the two operations. The multiplestage "with" (MSw) group was given training on the task during the interval between their two operations, as were Groups Sham, SSE, and SSL. The multiple-stage "without" (MSw/o) group did not receive training between the two unilateral lesions.

All animals received the first operation within 4 days of reaching criterion in original training. After the first operation, all animals were allowed 5 days of postoperative recovery. The animals in Groups Sham, SSE, SSL, and MSw were then given 4 days of interoperative training that was identical to original training. The animals that received no interoperative training (Group MSw/o) were kept in their home cages throughout this period but did receive free access to wet mash for $100 \mathrm{sec}$ per day. After the interoperative interval, the animals were given the second operation and 5 days of postoperative recovery; they were then retrained to criterion using the same procedures and criterion as in original training.

\section{Surgical Procedures}

Lesions were made by aspiration under sodium pentobarbital anesthesia, and encompassed striate and peristriate cortex [Krieg's (1946) areas: 17, 18, 18a]. The bilateral lesions were made either in one operation or in two unilateral operations, depending upon group designation in a manner identical to that described in Scheff et al. (1976).

\section{Electrophysiological Procedures}

After retraining, five animals from each group were mapped. The animals were anesthetized with alpha chloralose $(125 \mathrm{mg} / \mathrm{kg})$, placed in a head holder, and either the left or the right hemisphere was exposed. The dura was left intact, and saline heated to $37^{\circ} \mathrm{C}$ was applied to keep the dura from drying out. The rectal temperature of the subjects was monitored periodically. Only one hemisphere was explored while both eyes were stimulated with light flashes from a Grass PS-5 strobe at a rate of $0.25 \mathrm{~Hz}$. Each eye was prepared for stimulation by removing the lid and applying a thin layer of mineral oil to protect the cornea from drying.

Evoked potentials were recorded from the surface of the intact dura with a stainless steel monopolar electrode $0.25 \mathrm{~mm}$

Table 1

Summary of Design and Group Mean Errors to Criterion in Original Training and Retraining

\begin{tabular}{|c|c|c|c|c|c|c|}
\hline \multirow[b]{2}{*}{ Group } & \multirow{2}{*}{$\frac{\text { Train to Criterion }}{\text { Mean Errors }}$} & \multirow[b]{2}{*}{ Operation 1} & \multirow{2}{*}{$\begin{array}{c}\text { 4-Day Inter Op } \\
\text { Interval }\end{array}$} & \multirow[b]{2}{*}{ Operation 2} & \multirow{2}{*}{$\frac{\text { Retrain to Criterion }}{\text { Mean Errors }}$} & \multirow{2}{*}{$\begin{array}{c}\text { Electro- } \\
\text { physiology }\end{array}$} \\
\hline & & & & & & \\
\hline Sham & 52.5 & Sham & Train & Sham & 3.2 & MAP \\
\hline SSE & 58.5 & Bilat V & Train & Sham & 64.5 & MAP \\
\hline SSL & 47.2 & Sham & Train & Bilat V & 54.5 & MAP \\
\hline MSw/o & 52.0 & Unilat V & No Training & Unilat V & 41.5 & MAP \\
\hline MSw & 51.5 & Unilat V & Train & Unilat V & 17.1 & MAP \\
\hline
\end{tabular}

Note-Abbreviations: $S S E$ = single stage (lesion) early, $S S L=$ single stage late, $M S w / o=$ multiple stage (lesion) without interoperative training, $M S w=$ multiple stage with interoperative training, Bilat $V=$ bilateral lesion of visual cortex (Krieg's areas $17,18,18 a)$, Unilat $V=$ unilateral lesion of visual cortex. 
in diameter. The indifferent electrode was a small screw inserted into the nasal bone. The exploring electrode was moved in 1-mm steps to map the entire intact cortex posterior to bregma from 1.5 to $5.5 \mathrm{~mm}$ from midline.

A concentric bipolar electrode was lowered into the lateral geniculate [de Groot (1959) coordinates: A.P. -3.8, L. -3.6, V. -1.0 ] to monitor noncortical visual activity throughout the mapping procedure. All evoked potentials were amplified by Tektronix 3A9 differential amplifiers, averaged (CAT 1000) and drawn out on a X-Y plotter (Plotamatic). Twenty stimulus presentations were used for each average.

\section{Histological Procedures}

For histological verification of the lesions, the brains were perfused in situ via the left ventricle of the heart with physiological saline and $10 \%$ buffered Formalin solution. The whole brains were then photographed, and the photographic slides of the lesioned areas were projected onto Lashley diagrams and the extent of the lesions was traced.

The brains were sectioned on a freezing microtome at $40 \mu$, and every third section was saved through the lateral geniculate. The sections were stained with cresyl violet and mounted for microscopic examination.

\section{RESULTS}

\section{Anatomical Results}

Lasley diagrams representing the minimal and maximal lesions are shown in Figure 1. All of the lesions encompassed most of areas 17, 18, 18a. Extensive thalamic retrograde degeneration was found in most animals identical to that described in Scheff et al. (1976).

\section{Behavioral Results}

The mean number of errors to criterion in original training and retraining are shown in Table 1. Errors to criterion in training and retraining are graphically represented in Figure 2. A one-way analysis of variance was used to assess overall differences and Tukey's HSD test (Kirk, 1968) was used for multiple comparisons among means.

There were no group differences in original training $(p>.05)$. Groups SSE, SSL, and MSw/o made significantly more errors in retraining than either Group Sham or Group MSw ( $p<.01)$. Groups Sham and MSw did not differ from each other in retraining $(\mathrm{p}>$.05).
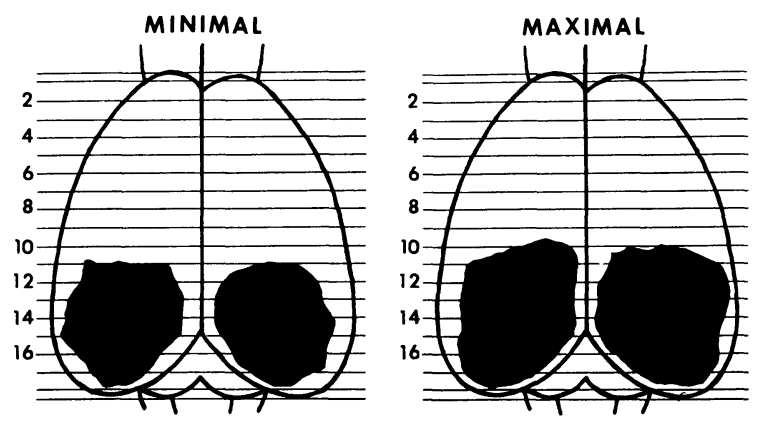

Figure 1. Lashley diagrams representing the minimal and maximal visual cortex lesions.

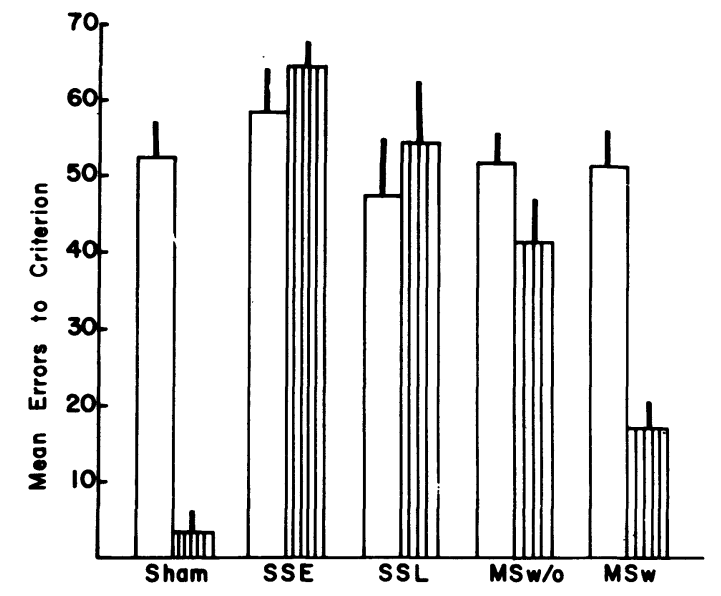

Figure 2. Group mean errors to criterion in training (white bars) and retraining (shaded bars). Vertical lines indicate standard error of the mean.

Within-groups comparisons of training and retraining errors to criterion showed no differences between these measures, i.e., no savings in retraining, for Groups SSE, SSL, and MSw/o. Both Group Sham and Group MSw showed positive savings with significantly fewer errors in retraining than in training $(\mathrm{p}<.01)$.

\section{Electrophysiological Results}

All evoked response averages were first scored blind by three independent judges for the presence of an evoked response in the first $120 \mathrm{msec}$ following stimulus onset. Seventy percent of the points mapped in Group MSw were judged to show evoked responses, while responses were judged present in only $39 \%$ of the points for Group MSw/o, 32\% for Group SSL, and $24 \%$ for Group SSE.

Peak-to-peak amplitudes of components in the evoked responses were also measured. The amplitudes were measured as the vertical distance between the positive and negative peaks of two adjacent components. Individual $t$ tests were performed to determine if the amplitude means were reliably different with respect to surgical group.

Group MSw differed significantly in amplitude from the other surgical groups: SSE $(t=4.841$, df $=9, \mathrm{p}<.001)$, SSL $(\mathrm{t}=2.862, \mathrm{df}=9, \mathrm{p}<.02)$. $\mathrm{MSw} / \mathrm{o}(\mathrm{t}=4.494, \mathrm{df}=9, \mathrm{p}<.01)$. Peak latencies did not differ among groups.

Representative responses are shown in Figure 3. In Column A, the responses were all recorded from the location (approximately $5 \mathrm{~mm}$ posterior to bregma, $4 \mathrm{~mm}$ lateral to midline) at which Groups SSE, SSL, and MSw/o were judged to show the greatest peak-to-peak amplitude. The responses in Column B were derived from the point (approximately $4 \mathrm{~mm}$ posterior to bregma, $5 \mathrm{~mm}$ lateral to midline) at which Group MSw was judged to show the greatest peak-to-peak amplitude. Representative lateral 


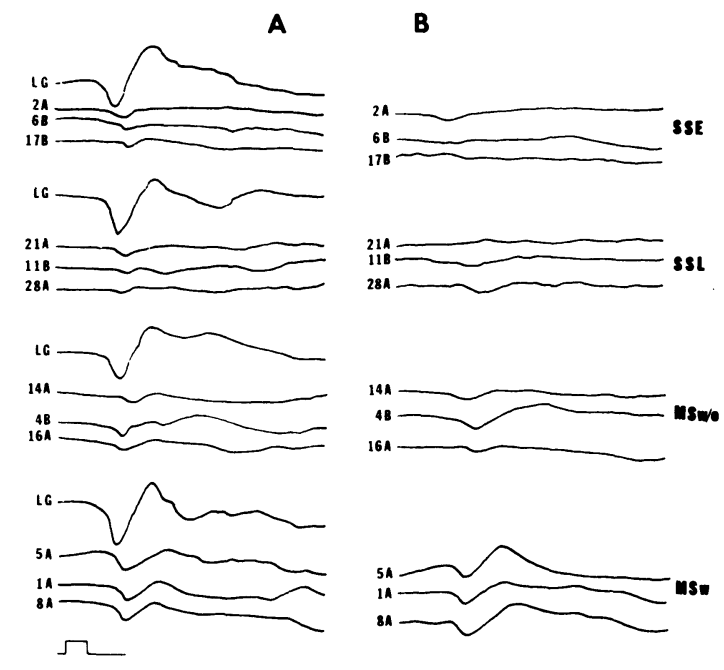

Figure 3. Representative geniculate responses and cortical responses from each group. One typical lateral geniculate response is shown for each lesion group in Column $A$ and is labeled "LG." Cortical responses from individual subjects in each group are labeled as $2 \mathrm{~A}, \mathbf{6 B}, \ldots$ etc. The cortical responses in Column $A$ all derive from a point $5 \mathrm{~mm}$ posterior to bregma, $4 \mathrm{~mm}$ lateral to midline. Those in Column B, from a point $4 \mathrm{~mm}$ posterior to bregma, $5 \mathrm{~mm}$ lateral to midline. Calibration mark: 20 msec $\times 20$ microV.

geniculate (LG) responses for each group are shown in Column A. The dark area in Figure 4 represents recording sites for Column $A$ and the hatched area represents recording sites for Column $B$.

\section{DISCUSSION}

Animals that received MS lesions of visual cortex in conjunction with IOI training showed positive savings on a black-white appetitive discrimination. Animals that received SS lesions and animals that received MS lesions with no IOI training did not show savings. This confirms previous demonstrations of the serial lesion effect for shock escape/ avoidance learning (Glendenning, 1972; Petrinovich \& Carew, 1969; Thompson, 1960).

The present results are difficult to reconcile with the notion that the postoperative performance differences between animals with SS lesions and those with MS lesions can be accounted for primarily on the basis of difference in surgical trauma. The two SS-lesion groups did not differ in postoperative performance, even though the SSE animals had 9 additional days to recover from the lesion trauma. More importantly, Group MSw/o also showed no savings in retraining while Group MSw did not differ from Group Sham in postoperative performance. When surgical trauma is equated but IOI experience is not, the serial lesion effect is present only for those MS animals that receive IOI training.
The reasons why IOI training plays a crucial role in the serial lesion effect remain obscure. The results of Dru, Walker, and Walker (1975) suggest that training per se may not be necessary. In their experiment, rats with MS visual cortex lesions that were allowed to freely explore a complex visual environment during the IOI were able to relearn a pattern discrimination, but MS animals that received passive visual exposure during the IOI were not. In the present experiment, the animals in Group MSw/o spent the IOI in the home cage and may have been exposed to "passive" visual experience while the animals of Group MSw were required to coordinate visual and motor activity while running in the T-maze during the IOI. It remains for future research to demonstrate whether IOI training is itself necessary for the serial lesion effect in a brightness task or whether such training merely provides a convenient method for ensuring that the MS animal will experience integrated visual-motor activity during the IOI.

The effects of additional cortical lesions on discrimination learning are markedly different in animals with SS and MS visual cortex lesions. Animals with prior SS visual cortex lesions show positive savings following additional cortical lesions (Horel et al., 1966; Lashley, 1922; Scheff et al., 1976). Animals with prior MS visual cortex lesions show negative savings following additional cortical lesions but only if the additional lesion damages cortex contiguous with the visual cortex lesion site (Scheff et al., 1976). Brightness learning following SS visual cortex lesions is virtually identical in animals with or without prior lesions of most of the nonvisual cortex (Horel et al., 1966). Animals show positive savings following MS visual cortex lesions that have been

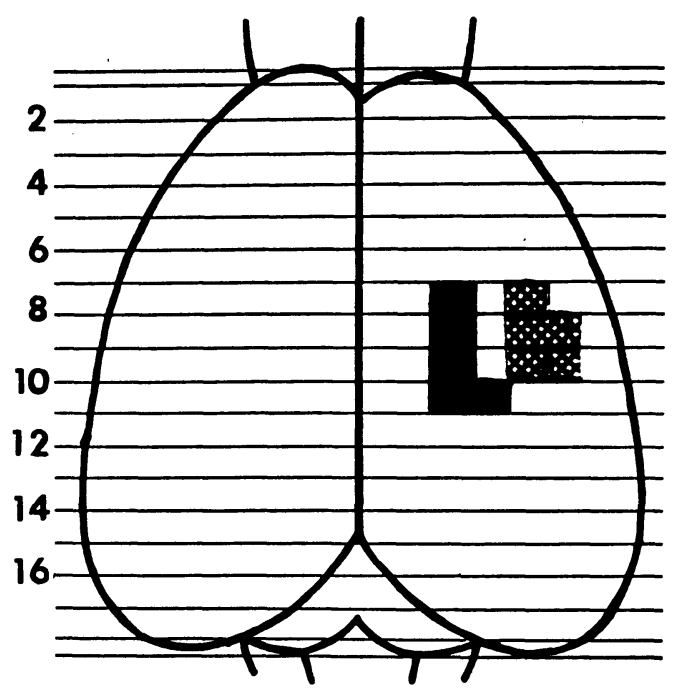

Figure 4. Cortical areas from which evoked-response peak-topeak amplitude is maximal for Group MSw (hatched) or for Groups SSE, SSL, and MSw/o (dark). 
preceded by lesions of cortex not contiguous with the visual cortex, but show negative savings following MS visual cortex lesion preceded by lesions of cortex contiguous with the visual cortex (Scheff et al., 1976).

In the present study, the remaining neocortex adjacent to the visual cortex lesions was examined electrophysiologically. The results indicate that the adjacent cortex in Group MSw responds quite differently from that in the other lesioned groups, with more points judged to be responsive with greater peak-to-peak amplitudes. Coupled with earlier findings, these results suggest that the adjacent cortex is differently organized for processing visually mediated responses in animals that show the serial lesion effect.

\section{REFERENCES}

DE GROOT, J. The rat forebrain in stereotaxic coordinates. Amsterdam: Noord-Hollandische, 1959.

Dru, D., WAlker, J. P., \& W Alker, J. B. Self-produced locomotion restores visual capacity after striate lesions. Science, 1975, 187, 265-266.

GellermanN, L. W. Chance orders of alternating stimuli in visual discrimination experiments. Journal of Genetic Psychology, 1933, 42, 206-208.

GLendenNing, R. L. Effects of training between two unilateral lesions of visual cortex upon ultimate retention of black-white discrimination habits by rats. Journal of Comparative and Physiological Psychology, 1972, 80, 216-229.

Horel, J. A., Bettinger, J. A., Royce, G. J., \& Meyer, D. R. Role of neocortex in the learning and relearning of two visual habits by the rat. Journal of Comparative and Physiological Psychology, 1966, 67, 66-78.
Kircher, K. A., Braun, J. J., Meyer, D. R., \& Meyer, P. M. Equivalence of simultaneous and successive neocortical ablations in production of impairments of retention of black-white habits in rats. Journal of Comparative and Physiological Psychology, 1970, 71, 420-425.

KIRK, R. E. Experimental design: Procedures for the behavioral sciences. Belmont, Calif: Wadsworth, 1968.

KRIEG, W. J. S. Connections of the cerebral cortex: I. The albino rat. Journal of Comparative Neurology, 1946, 84, 221-275.

LAshley, K. S. Studies of cerebral function in learning. Psychobiology, 1920, 2, 55-135.

LASHLEY, K. S. Studies of cerebral function in learning. IV. Vicarious function after destruction of the visual areas. American Journal of Physiology, 1922, 59, 44-71.

LASHLEY, K. S. Studies of cerebral function in learning. VII. The relation between cerebral mass, learning and retention. Journal of Comparative Neurology, 1926, 41, 1-58.

LASHLEY, K. S. Brain mechanisms and intelligence. Chicago: University of Chicago Press, 1929.

LASHLEY, K. S. The mechanism of vision. II. The influence of cerebral lesions upon the threshold of discrimination for brightness in the rat. Journal of Genetic Psychology, 1930, 37, 461-480.

Petrinovich, L., \& CAREw, T. J. Interaction of neocortical size and interoperative experience in retention of a learned brightness discrimination. Journal of Comparative and Physiological Psychology, 1969, 68, 451-454.

Scheff, S. W., Wright, D. C., Morgan, W. K., \& Powers, R. B. The differential effects of additional cortical lesions in rats with single or multiple stage lesions of the visual cortex. Physiological Psychology, 1977, 5, 97-102.

SPEAR, P. D., \& BARBAS, H. Recovery of pattern discrimination ability in rats receiving serial or one-stage visual cortex lesions. Brain Research, 1975, 94, 337-346.

Thомpson, R. Retention of a brightness discrimination following neocortical damage in the rat. Journal of Comparative and Physiological Psychology, 1960, 53, 212-215.

(Received for publication June 25, 1976; revision accepted November $16,1976$. 